\title{
Alternativas para el monitoreo en línea de soluciones poliméricas en procesos EOR
}

\author{
Blanca Johanna Báez Serrano; Diana Alejandra Montealegre Peña ${ }^{1 *}$; Rubén Hernán Castro García²; \\ Mario Ardila Moreno ${ }^{3}$; Andrés Felipe Suárez Barbosa ${ }^{1}$
}

doi: http://dx.doi.org/10.18273/revfue.v18n2-2020003 @c (i)

Forma de citar: Báez-Serrano, B. J., Montealegre-Peña, D. A., Castro-García, R. H., Moreno, M. A., \& Suárez-Barbosa, A. F. (2020). Alternativas para el monitoreo en línea de soluciones poliméricas en procesos EOR. Revista Fuentes, el reventón energético, 18(2), 45-56. https://doi.org/10.18273/revfue.v18n2-2020003

\section{Resumen}

La inyección de polímeros es un método de recobro mejorado utilizado en el mundo como tecnología exitosa, sin embargo, uno de los principales problemas es la sensibilidad de las soluciones poliméricas a la degradación térmica, química, mecánica y/o biológica. En ese sentido, el monitoreo de la viscosidad permite la toma de decisiones rápida durante la operación, con el fin de asegurar un proceso eficiente y así el incremento en el factor de recobro. En este trabajo se analizaron diferentes viscosímetros in-line para el monitoreo en tiempo real de la viscosidad de soluciones poliméricas. Adicionalmente, se realizó un análisis económico de los viscosímetros técnicamente factibles y disponibles mediante un sondeo de mercado, los cuales se compararon con la metodología de monitoreo empleada actualmente en Ecopetrol S.A.

Palabras clave: análisis técnico-financiero, inyección de polímero, viscosidad soluciones poliméricas, viscosímetro in-line, rangos operacionales, recobro mejorado.

\section{In-line monitoring alternatives of polymer solution viscosity in EOR process}

\begin{abstract}
Polymer injection is an improved recovery method widely used in the world as a successful technology; however, one of the main problems is polymer solutions sensitivity to thermal, chemical, mechanical and/or biological degradation. In this way, viscosity monitoring allows making decision quickly in the operation in order to ensure an efficient process and thus allowing an increase in the recovery factor. In this paper different in-line viscometers were analyzed for the real time monitoring of the polymer solutions viscosity. In addition, an economic analysis of the technically feasible and available viscometers was carried out through a market survey, which were compared with the current monitoring methodology used in Ecopetrol S.A.
\end{abstract}

Keywords: technical-financial analysis, polymer flooding, polymer solutions viscosity, in-line viscometer, operational ranges, enhanced Oil Recovery.

\footnotetext{
${ }^{1}$ Universidad de America. Avenida Circunvalar No 20-53. Bogotá, Colombia. (*) E-mail: dianalejam@gmail.com

${ }^{2}$ Instituto Colombiano del Petróleo (ICP). Vía Piedecuesta Km 7. Piedecuesta, Colombia

${ }^{3}$ Ecopetrol S.A. Cra. 13 No 36-24. Bogotá, Colombia
} 


\section{Introducción}

Teniendo en cuenta que el factor de recobro en Colombia es del 19\% (Jimenez, y otros, 2017), el mayor reto en la industria de los hidrocarburos es buscar alternativas que ayuden a incrementar el mismo. Con base en esto, desde el año 2014, en Colombia se han implementando procesos de inyección de polímero con el fin de lograr una mayor extracción del petróleo remanente existente en los yacimientos. La inyección de polímero es un proceso de recobro mejorado que consiste en incrementar la viscosidad del agua inyectada (Castro, y otros, 2016), (Molano, A. M. J., y otros, 2014), mejorando la relación de movilidad y generando un barrido más eficiente del yacimiento (Aya, C. L. D., y otros, 2018). Para aumentar la eficiencia del proceso de recobro, es necesario tener un control y monitoreo permanente de la viscosidad de la solución polimérica (Araujo, Y. C., \& Araujo, M., 2018).

En las facilidades de superficie, la solución polimérica está expuesta a diferentes factores que causan su degradación mecánica debido a mezcladores estáticos, bombas, líneas de inyección (especialmente si se presentan cambios bruscos de dirección de flujo y/o diametro), choques, accesorios, etc., presentes desde las instalaciones de preparación de la solución polimérica hasta la llegada a cabeza de pozo inyector (Araujo, Y. C., \& Araujo, M. 2018), (Pinto, M. S., y otros, 2018). Actualmente, en Ecopetrol S.A. se emplea una metodología para monitorear la viscosidad de la solución polimérica, la cual requiere tomar la muestra directamente en la tubería y después llevarla al laboratorio para su respectivo proceso de medición. En ese sentido, crece la necesidad de poder monitorear la viscosidad de las soluciones poliméricas en tiempo real, controlando la integridad del polímero a condiciones de superficie; para lograr esto, existen viscosímetros in-line y su selección técnica depende de las condiciones operacionales del campo en el cual se desee implementar.

En este trabajo, se realiza una descripción detallada de ocho viscosímetros in-line disponibles en el mercado y se evalúa la posibilidad de implementación en cinco pilotos de inyección de polímeros que se ejecutan en Colombia. La factibilidad técnica tiene en cuenta los parámetros operacionales, principios físicos, rangos operativos, ventajas y desventajas de los viscosímetros. Finalmente, se realiza un análisis financiero en uno de los pilotos con alto potencial de reservas e interés en la implementación de estas alternativas, comparando la metodología actual de monitoreo de viscosidad con la implementación de viscosímetros in-line (Báez y Montealegre, 2019).

\section{Metodología}

La metodología actual para el monitoreo de la viscosidad de las soluciones poliméricas, consiste en muestrear en la línea de inyección y posteriormente realizar la medición de viscosidad en un viscosímetro en laboratorio. Como se puede observar en la figura 1, las muestras de solución polimérica son tomadas en cabeza de pozo con un sistema especial de tomamuestra, usando un montaje que evita la degradación mecánica de la solución durante el muestreo.

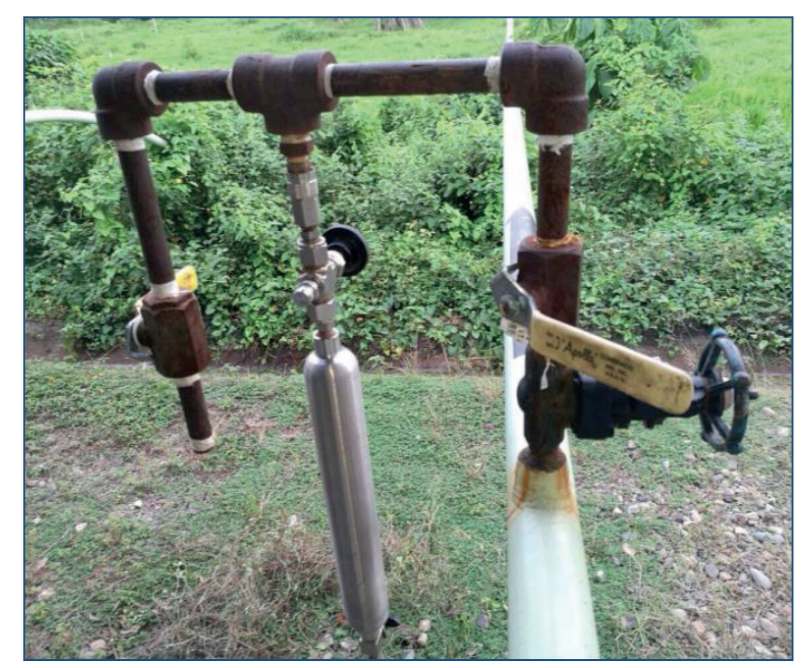

Figura 1. Toma muestra de solución polimérica en campo.

Posteriormente, se realiza la medición de la solución polimérica usando el viscosímetro Brookfield como se muestra en la figura 2, este viscosímetro es de tipo rotacional y permite tener mediciones de viscosidad a diferentes esfuerzos de corte. Para esta medición, es necesario ajustar la temperatura de la muestra mediante un baño termostático, calibrar, inicializar y finalmente asegurar el resultado obtenido.

En la etapa de factibilidad experimental fluidofluido realizado en el laboratorio (Ecopetrol S.A. Instituto Colombiano del Petróleo, 2016), se realizan diferentes ensayos con el fin de evaluar y determinar las propiedades de la solución polimérica, la figura 3 resume dichos ensayos experimentales. 


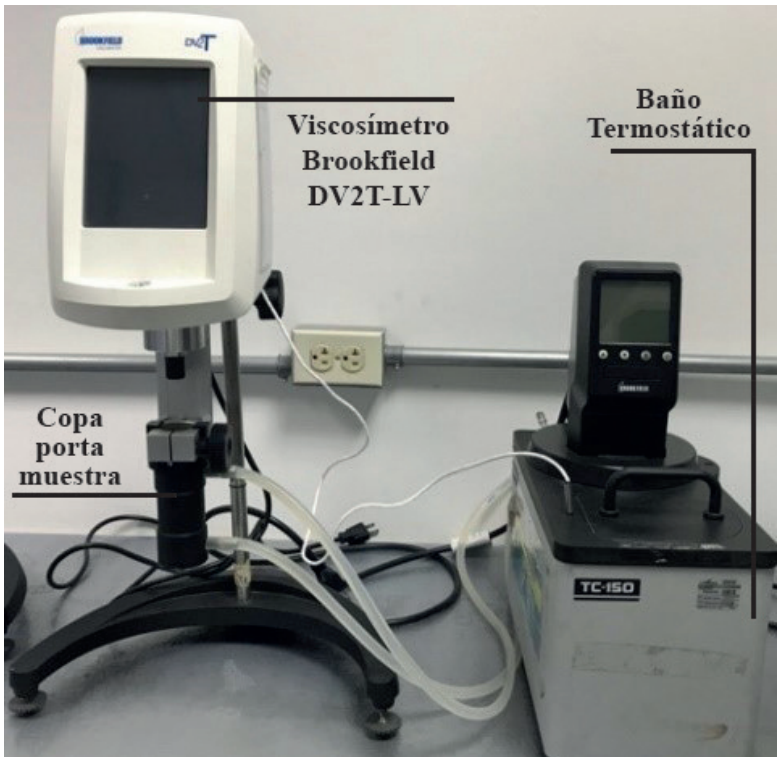

Figura 2. Equipo de medición de viscosidad, viscosímetro Brookfield DV2T-LV.
Por otro lado, en la etapa de factibilidad experimental roca-fluido evalúa la adsorción del polímero y volumen poroso no accesible (IPV) de las soluciones a condiciones de saturación de agua $100 \%(\mathrm{Sw} 100 \%)$ y saturación de aceite residual (Sor). Adicionalmente, se determina el Factor de Resistencia (RF) para evaluar la reducción de la movilidad y finalmente el Factor de Resistencia Residual (RRF) que permite determinar la reducción de la permeabilidad.

Es importante precisar que en el momento de realizar el monitoreo a condiciones de superficie se debe tener en cuenta principalmente los resultados de factibilidad experimental fluido-fluido. Fundamentalmente se debe monitorear la viscosidad de la solución polimérica (madre y diluida) para el aseguramiento y control de calidad ya que es una de las principales propiedades que afectan la eficiencia del proceso de recobro.

Análisis de espectroscopia (caracterización del polímero)

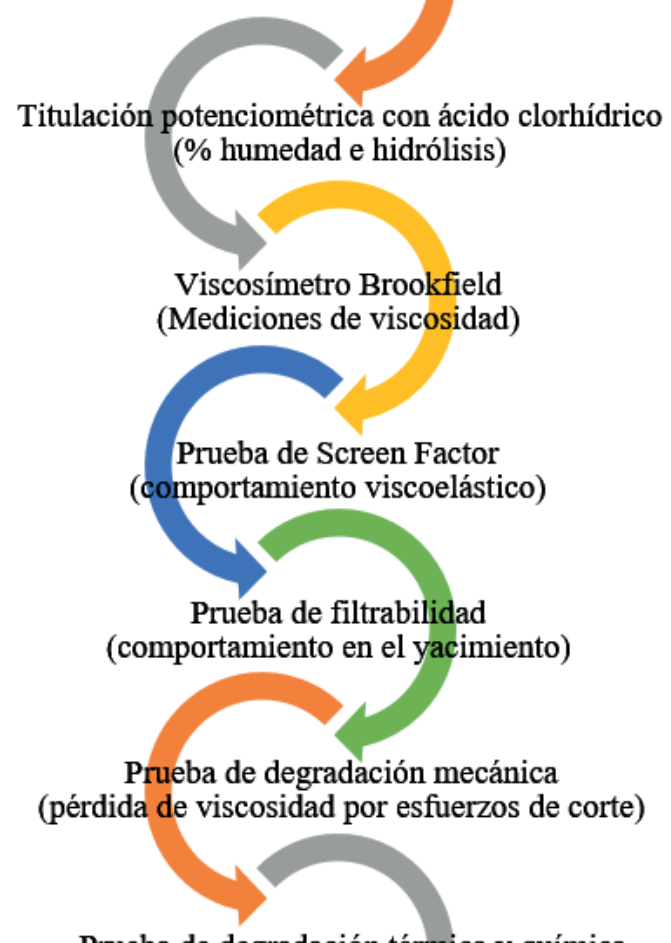

Prueba de degradación térmica y química

Figura 3. Factibilidad experimental fluido-fluido. 


\section{Viscosímetros in-line}

En la tabla 1, se genera una clasificación de los viscosímetros en línea de acuerdo con su principio operacional.

\section{Viscosímetro in-line A1}

Cuenta con un sensor de viscosidad el cual funciona con tecnología de vibración resonante. Adicionalmente, cuenta con una varilla vibrante ubicada en el sensor, la cual es mantenida en oscilación por corriente eléctrica y puede medir la viscosidad dinámica de cualquier fluido industrial que tenga hasta una viscosidad de 1000000 cP. La electrónica del equipo permite entregar mediciones de temperatura y viscosidades en tiempo real, también genera información complementaria, gráficos y una función de seguridad que consiste en realizar mediciones de viscosidad a condiciones limites sin dañar el viscosímetro.

Tabla 1. Principios operacionales de los viscosímetros in-line.

\begin{tabular}{|c|c|c|}
\hline Principio & Descripción & Viscosímetro in-line \\
\hline Vibración & $\begin{array}{l}\text { Consisite en la vibración, torsión o traslado de un módulo sumergido en } \\
\text { el líquido, cuenta con un sensor cuyo tiempo de respuesta es muy corto } \\
\text { (casi nulo), generando que las variaciones de viscosidad se presenten } \\
\text { de manera continua, lo que permite controlar un proceso en presencia } \\
\text { de perturbaciones rápidas. }\end{array}$ & $\mathrm{A} 1, \mathrm{~A} 2$ y $\mathrm{B} 1$ \\
\hline Péndulo de torsión & $\begin{array}{l}\text { Se basa en la vibración rotacional de un péndulo de torsión sumergido } \\
\text { en un líquido, este péndulo se acopla a un disco y mide el número de } \\
\text { oscilaciones del mismo con el fin de calcular la viscosidad con los } \\
\text { cambios en su movimiento de rotación. }\end{array}$ & $\mathrm{C} 1$ \\
\hline Horquilla Vibrante & $\begin{array}{l}\text { Consiste principalmente en una horquilla de dos clavijas, las cuales } \\
\text { van vibrando a su frecuencia natural cuando se encuentran en el aire, } \\
\text { esta variable cambia según el medio en el cual se sumerja, cuando el } \\
\text { líquido cubre las horquillas, la frecuencia cae, permitiendo el cálculo } \\
\text { de viscosidad. }\end{array}$ & D1 \\
\hline $\begin{array}{c}\text { Presión dinámica del } \\
\text { fluido }\end{array}$ & $\begin{array}{l}\text { Consta de un tubo parcialmente abierto con un eje cilíndrico giratorio } \\
\text { en el interior, generado un espacio entre ellos por el cual circula el } \\
\text { fluido generando que este espacio se haga cada vez más pequeño y } \\
\text { que la pared del tubo se desvié por la presión del líquido. Un sensor } \\
\text { eléctrico mide la longitud de la deflexión y lo usa para calcular el valor } \\
\text { de viscosidad. }\end{array}$ & E1 y E2 \\
\hline Caída de presión & $\begin{array}{l}\text { Funciona con el principio de medición de la caída de presión a través de } \\
\text { un tubo calibrado a caudal constante, el sistema calcula la viscosidad } \\
\text { actual de la solución polimérica que pasa a través del viscosímetro } \\
\text { empleando una curva extrapolada que tiene en cuenta sus puntos de } \\
\text { calibración. }\end{array}$ & $\mathrm{F} 1$ \\
\hline
\end{tabular}

\section{Viscosímetro in-line A2}

Dispositivo de tipo vibrante, el cual cuenta con un diseño que permite integrarse fácilmente a procesos como extrusión de plásticos, polímeros o elastómeros, lo que permite la medición directa de la viscosidad dinámica del fluido en tiempo real evitando desfases fuera del proceso.

\section{Viscosímetro in-line B1}

Trabaja con la tecnología de vibración torsional, mediante un sensor sin partes móviles ni sellos. Este tipo de viscosímetros in-line puede acoplarse directamente en el proceso sin alteraciones de tuberías, proporciona medidas de viscosidad en tiempo real y se puede utilizar en ambientes ruidosos, de altas y bajas temperaturas. 


\section{Viscosímetro in-line C1}

Es empleado para la medición de densidad, viscosidad y temperatura del fluido, especialmente del aceite combustible. Emplea un sensor que emite una señal y su medición se puede usar en cálculos de flujo másico, la señal de salida de densidad puede ser conectada a una pantalla o computadora de flujo. Los componentes del sistema son sensor, carcasa y caja de conexiones.

\section{Viscosímetro in-line D1}

Dispositivo para la medición de la viscosidad multivariable de alto rendimiento, el cual permite un constante control y monitoreo de la viscosidad, densidad y temperatura del fluido. Su material es acero inoxidable con una carcasa en el transmisor, la cual es opcional para garantizar mayor resistencia a la corrosión. Su diseño de inserción es directo para tuberías de longitudes de hasta 4 metros, sistemas de derivación y tanques. El viscosímetro D1 esta compuesto por un transmisor integrado con interfaz local del operador opcional, una conexión de proceso, las puntas vibrantes y un detector de temperatura resistiva.

\section{Viscosímetro in-line E1}

Se ubica directamente dentro de la tubería por la cual pasa el fluido que se desee monitorear, arroja lecturas continuas de viscosidad y temperatura permitiendo el control de estas propiedades en tiempo real. El viscosímetro E1 es empleado en la industria de los hidrocarburos como lubricantes, mezcla de asfalto con polímero, adhesivos de almidón, suspensiones y otros líquidos de proceso.

\section{Viscosímetro in-line E2}

Permite monitorear la viscosidad del fluido durante su paso por la tubería en superficie a la temperatura del proceso, cubre un rango de viscosidad de 1 a $50000 \mathrm{cP}$. Este viscosímetro se sumerge directamente en el fluido y es empleado en diferentes líquidos de procesos como fluidos petroquímicos, lubricantes, suspensiones, detergentes, adhesivos de almidón, restos de cerámica, entre otros.

A medida que el fluido pasa por el sensor, este va registrando la viscosidad y temperatura, con el fin de tener un control de 24 horas. El diseño permite su aplicación en zonas de alto riesgo, previniendo chispas y generando un proceso confiable.

\section{Viscosímetro in-line F1}

Es usado principalmente en la industria petrolera. Se ha empleado en la medición de soluciones poliméricas, permitiendo medidas estables y aproximadas sin degradar la solución. Este equipo se ubica en una conexión by-pass de la línea principal por donde fluye la solución.

\section{Resultados}

En la tabla 2, se especifican los parámetros operativos más importantes de los viscosímetros in-line.

Tabla 2. Rangos operacionales de los viscosímetros in-line.

\begin{tabular}{|c|c|c|c|c|c|c|c|c|}
\hline $\begin{array}{c}\text { Viscosímetro } \\
\text { in-line } \\
\text { Parámetro }\end{array}$ & A1 & A2 & B1 & C1 & D1 & E1 & $\mathbf{E 2}$ & F1 \\
\hline Viscosidad (cP) & $0,1-1000000$ & $1-10000000$ & $0-10^{\wedge} 9$ & $0-50$ & $0,5-12500$ & $1-50000$ & $1-50000$ & $1-1000$ \\
\hline $\begin{array}{c}\text { Temperatura } \\
\left({ }^{\circ} \mathbf{F}\right)\end{array}$ & $32-390$ & $32-662$ & $-58-302$ & $32-392$ & $-58-392$ & $23-392$ & $23-383$ & $41-176$ \\
\hline Presión (psi) & $0-870$ & $0-5076$ & $0-10000$ & $0-580$ & $1450-3000$ & $0-362$ & $0-362$ & $>2900$ \\
\hline Densidad (g/cc) & $0,6-1,6$ & N.A. & N.A. & $0,75-1,1$ & $0.6-3$ & N.A. & N.A. & N.A. \\
\hline
\end{tabular}


Basados en sus rangos operacionales, se pueden realizar las siguientes observaciones:

- El viscosímetro E1 no se ve afectado por las caídas de presión, por otro lado, las lecturas proporcionadas por el dispositivo pueden ser leídas directamente en el instrumento o en un tablero instalado a una distancia hasta de 250 metros.

- El viscosímetro D1 cuenta con una mayor precisión cuando se calibra para trabajar a viscosidades menores a $10 \mathrm{cP}$.

- Los viscosímetros C1 y F1 únicamente se pueden emplear para monitorear la solución final después de pasar por la etapa de dilución, debido a que su rango de operación no alcanza el valor requerido para determinar la viscosidad de la solución madre del proceso de Inyección de polímero.

- El Viscosímetro $\mathrm{C} 1$ es el viscosímetro más limitado para el monitoreo de viscosidad debido a que opera hasta $50 \mathrm{cP}$.
- Todos los dispositivos cumplen con el rango de operación de temperatura debido a que, al ser instalado en superficie, se manejan temperaturas cercanas a la temperatura ambiente.

- Dependiendo de las condiciones operativas de inyección, se puede seleccionar el viscosímetro que cumpla con el rango de operación de presión de inyección para garantizar su correcto funcionamiento.

- Los viscosímetro A1, C1 y D1 ofrecen la opción adicional de medición de densidad.

Para la selección de un viscosímetro in-line adecuado, es necesario tener conocimiento de las variables que se manejan, ya que identificarlas de manera adecuada permitirá realizar el monitoreo de la solución polimérica en tiempo real conservando la integridad de la solución polimérica. En ese sentido, en la tabla 3 , se presenta la calidad de agua y las condiciones operacionales en cada piloto de inyección de polímero.

Tabla 3. Condiciones operacionales de los pilotos de inyección de polímero.

\begin{tabular}{|c|c|c|c|c|c|c|c|}
\hline Piloto & $\begin{array}{c}\text { Agua de } \\
\text { preparación }\end{array}$ & $\begin{array}{c}\text { Cloruros } \\
(\mathrm{mg} / \mathrm{l})\end{array}$ & Hierro & $\begin{array}{l}\text { Grasas y } \\
\text { aceites }\end{array}$ & $\begin{array}{c}\text { Viscosidad } \\
\text { solución final } \\
\text { (cP) }\end{array}$ & $\begin{array}{c}\text { Presión de } \\
\text { superficie } \\
\text { (psi) }\end{array}$ & $\begin{array}{c}\text { Caudal } \\
\text { inyectado } \\
\text { (BPD) } \\
\end{array}$ \\
\hline 1 & Fresca & $<50$ & $\begin{array}{c}\text { Cumple } \\
\text { especificación }\end{array}$ & $\begin{array}{c}\text { Cumple } \\
\text { especificación }\end{array}$ & 20 & $2800-3100$ & $1000-2000$ \\
\hline 2 & Fresca & $<50$ & $\begin{array}{c}\text { Cumple } \\
\text { especificación }\end{array}$ & $\begin{array}{c}\text { Cumple } \\
\text { especificación }\end{array}$ & 11 & $1600-2200$ & $300-700$ \\
\hline 3 & $\begin{array}{l}\text { Producción / } \\
\text { fresca }\end{array}$ & $\begin{array}{l}4500 / \\
<100\end{array}$ & $\begin{array}{c}\text { Fuera de } \\
\text { especificación }\end{array}$ & $\begin{array}{c}\text { Fuera de } \\
\text { especificación }\end{array}$ & 3,3 & $1800-2200$ & $1500-3000$ \\
\hline 4 & Producción & 2000 & $\begin{array}{c}\text { Fuera de } \\
\text { especificación }\end{array}$ & $\begin{array}{c}\text { Fuera de } \\
\text { especificación }\end{array}$ & 30 & $0-150$ & $5000-6500$ \\
\hline 5 & Fresca & $<50$ & $\begin{array}{c}\text { Cumple } \\
\text { especificación }\end{array}$ & $\begin{array}{c}\text { Cumple } \\
\text { especificación }\end{array}$ & $25-40$ & $1600-1800$ & $600-1000$ \\
\hline
\end{tabular}

Variables que afectan la integridad de la solución polimérica

La calidad del agua es fundamental para garantizar la viscosidad requerida en la solución polimérica. Por esto, es necesario analizar la fuente y el tipo de agua que se esté empleando, ya que se si se dispone del agua de producción, ésta puede contener impurezas que afecten las cadenas del polímero causando una viscosidad menor a la diseñada.

La calidad de agua empleada en los pilotos de inyección de polímero se infieren al analizar los resultados del diagnóstico de seguimiento de parámetros fisicoquímicos del agua de preparación. La caracterización fisicoquímica se realiza mediante la medición de los siguientes parámetros: hierro, $\mathrm{pH}, \mathrm{H}_{2} \mathrm{~S}, \mathrm{O}_{2}$, y $\mathrm{CO}_{2}$ disueltos, grasas y aceites y turbidez (Ecopetrol S.A., 2018). La tabla 4 presenta valores aproximados de los parámetros de control fisicoquímicos del agua de preparación de los cinco pilotos de inyección de polímero; a su vez, se especifican los rangos recomendados por la literatura.

Para determinar el dispositivo adecuado para cada piloto de inyección, se comparan las condiciones del campo con los rangos de operación que garantizan un óptimo funcionamiento de los viscosímetros in-line. 
En ese sentido, las variables analizadas para el proceso de inyección de polímero fueron rango de medición de viscosidad y presión.

En la gráfica 1 se puede visualizar detalladamente la distribución de los viscosímetros según sus rangos de viscosidad y presión.
Posteriormente, en la tabla 5 se comparan las condiciones operacionales de cada piloto de inyección de polímero con los rangos operacionales de los viscosímetros in-line, para determinar que dispositivo se podría instalar eventualmente en cada piloto sin afectar las lecturas proporcionadas por los mismos y generar confiabilidad en el proceso.

Tabla 4. Parámetros fisicoquímicos del agua de preparación de los pilotos de inyección.

\begin{tabular}{|c|c|c|c|c|c|c|c|c|}
\hline Pilotos & $\begin{array}{c}\mathrm{Fe}^{++} \\
(\mathrm{ppm})\end{array}$ & $\begin{array}{l}\text { Cloruros } \\
\text { (ppm) }\end{array}$ & pH & $\begin{array}{c}\mathrm{H}_{2} \mathrm{~S} \\
(\mathrm{ppm})\end{array}$ & $\begin{array}{c}\mathrm{O}_{2} \\
(\mathrm{ppb})\end{array}$ & $\begin{array}{c}\mathrm{CO}_{2} \\
(\mathrm{ppm})\end{array}$ & $\begin{array}{c}\text { Grasas } \\
\text { y aceites } \\
(\mathbf{p p m})\end{array}$ & $\begin{array}{c}\text { Turbidez } \\
\text { (NTU) }\end{array}$ \\
\hline $\begin{array}{c}\text { Niveles } \\
\text { recomendados }\end{array}$ & $<1$ & $<\mathbf{5 0}$ & $6,5-8,5$ & 0 & $<20$ & $<10$ & $<5$ & $<2$ \\
\hline 1 & 0,1 & 10,01 & 7,73 & $\mathrm{ND}^{*}$ & ND & $<10$ & ND & 0,52 \\
\hline 2 & 0,1 & 20 & 7,88 & ND & ND & $<10$ & ND & 0,42 \\
\hline 3 & 1 & 15,9 & 6,82 & ND & 10 & 18 & 0,7 & 11,8 \\
\hline 4 & 3 & 1796 & 7,02 & 0,1 & ND & 16 & 17,63 & $\mathrm{NR}^{* *}$ \\
\hline 5 & ND & 20,2 & 7,65 & ND & 5000 & $<10$ & ND & 1,46 \\
\hline
\end{tabular}

*ND: no detectado

**NR: no realizado

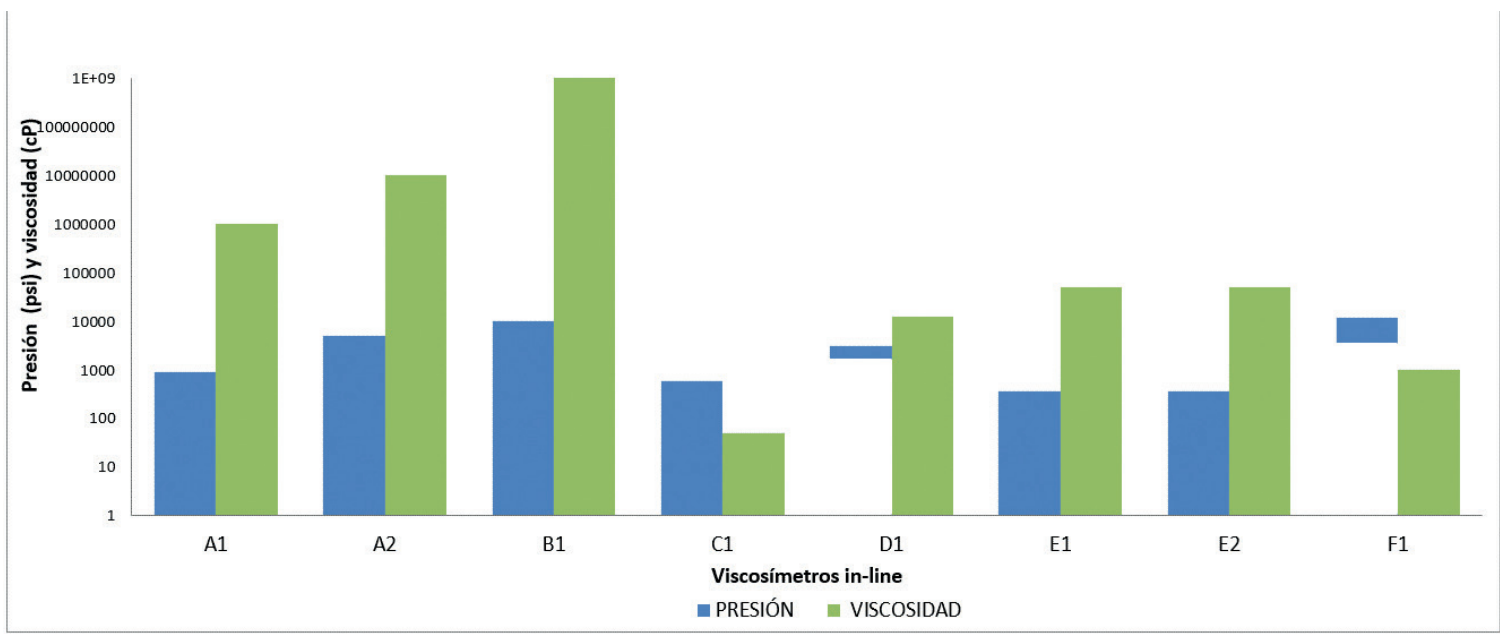

Gráfica 1. Rangos de presión y viscosidad de los viscosímetros in-line.

Tabla 5. Viscosímetros que cumplen condiciones operativas para los pilotos de Inyección polímero.

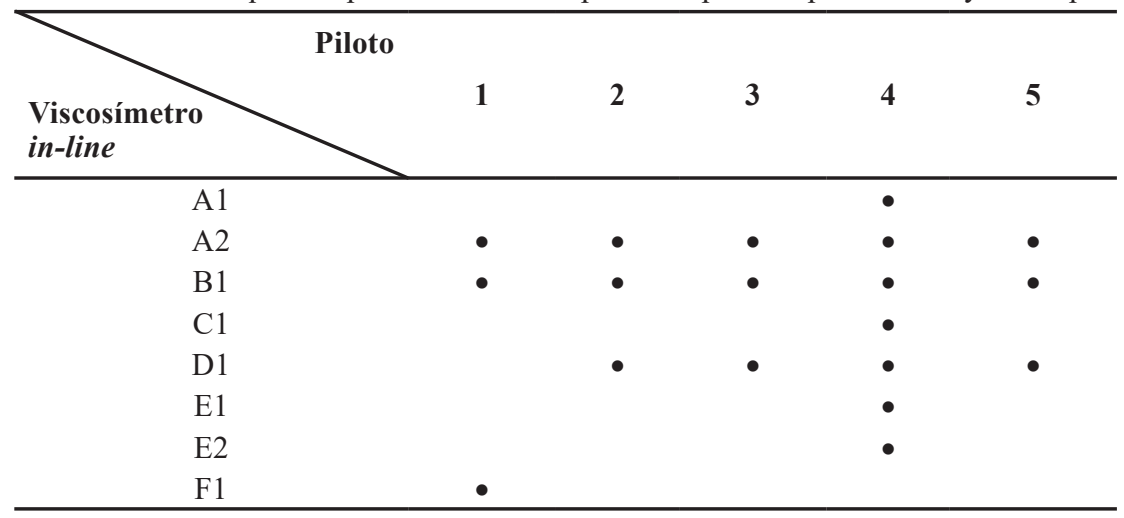


Teniendo en cuenta el análisis técnico realizado a los viscosímetros in-line, se determina que los viscosímetros A2 y B1 de tipo vibrante se ajustan a los rangos operacionales de todos los pilotos de inyección de polímeros ejecutados actualmente por Ecopetrol S.A.

Por otro lado, se determina que en el piloto 4 se podrían evaluar al mismo tiempo y mediante una prueba tecnológica el funcionamiento, precisión y aplicabilidad en la medición de soluciones poliméricas de la mayoría de viscosímetros.

\section{Análisis financiero}

Para la evaluación financiera se implementó una metodología desarrollada para proyectos de recobro mejorado (Guardia, V. M. D., y otros, 2011), se utilizó información de yacimientos y financiera de Ecopetrol S.A. En el análisis comparativo se seleccionó el piloto 4 mencionado en la sección anterior y los viscosímetros in-line E1 y E2 que tienen el principio de Presión dinámica del fluido.

Horizonte de Vida del Proyecto. 15 años.

Tasa de Descuento. Según Ecopetrol S.A., la tasa de descuento empleada en la evaluación del proyecto se definió al 10\% anual.

Precio del Crudo. Según Ecopetrol S.A., el precio por barril de crudo para el análisis, parte de un precio de referencia Brent de \$50 USD/Bbl.

Producción incremental. En la gráfica 2 se presenta la gráfica de producción incremental obtenida mediante simulación numérica de yacimientos, producto del eventual proceso de inyección de polímero en un patrón del campo correspondiente al piloto 4, correspondiente a 3,13 Millones de barriles de petróleo.

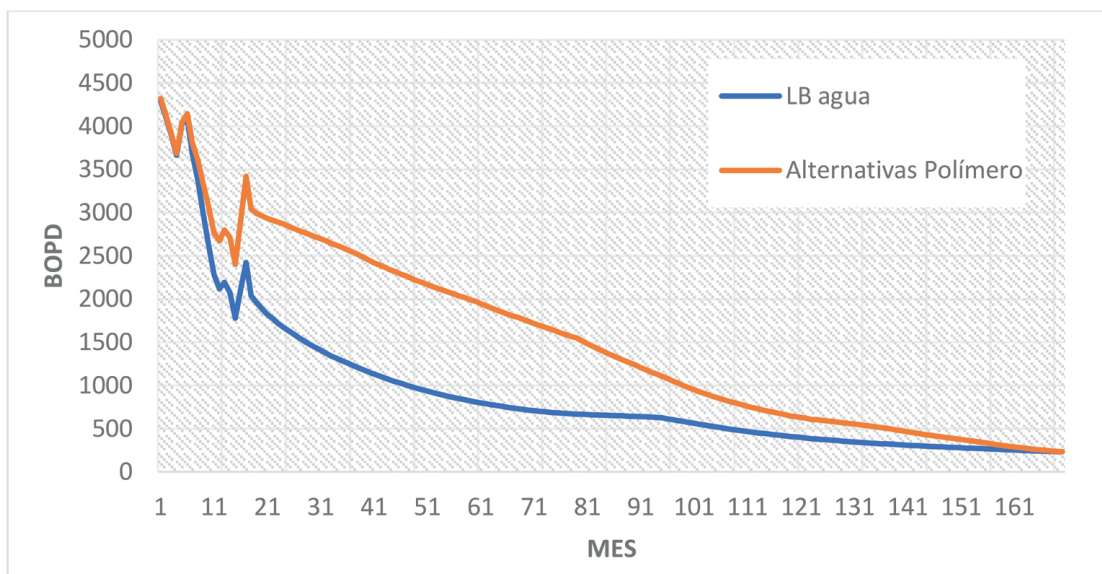

Gráfica 2. Curva de producción incremental con el proceso de inyección de polímero.

CAPEX. Se asume que el campo ya cuenta con las facilidades de inyección de agua, los pozos, completamiento y patronamiento adecuado. Adicionalmente, no se requieren modificaciones en la planta de inyección de polímero ni en el plan de manejo ambiental del campo. Los costos de CAPEX para la realización del flujo de caja del proyecto incluyen los costos de gerenciamiento, un workover para la conversión del pozo productor a inyector y por último unos algunos costos adicionales de contingencia.

OPEX. En cuanto a la metodología actual empleada para el monitoreo de la viscosidad de la solución polimérica, se tuvo en cuenta los monitoreos mensuales que se realizan en el piloto 4 , por otro lado, para el caso del análisis de los viscosímetros in-line, se incluyó la inversión y su instalación. Adicionalmente, se contempla el monitoreo mediante un ILT anual por pozo inyector, y un PLT cada 3 años por pozo productor, una estimulación al pozo inyector cada 3 años y costos de energía, sujetos al tamaño de las facilidades de cada tecnología.

Metodología actual. En la evaluación financiera de la metodología actual, la inversión incluye: Facilidades convencionales para la preparación de la solución polimérica con su correspondiente operación y mantenimiento, la concentración de polímero de 2300 ppm (la cual debe alcanzar el objetivo de $15 \mathrm{cP}$ en fondo de pozo) y costos de 65.096 USD/año, generados en la medición de la viscosidad, incluyendo gastos de control y calidad, recurso humano y elementos de protección personal. 
Para los costos asociados al OPEX de la metodología actual se tuvo en cuenta el costo del monitoreo de la viscosidad, cuyo costo anual fue 65.096 USD por año, estos únicamente se evalúan a 7 años ya que la duración de la inyección de polímero. En la tabla 6, se muestran los resultados obtenidos en el análisis de la metodología actual.

Tabla 6. Resumen resultados metodología actual.

\begin{tabular}{lc}
\hline VPN (USD) & $\mathbf{1 . 3 5 1 . 1 0 7}$ \\
\hline PRODUCCIÓN INCREMENTAL (Bbls) & 3.136 .367 \\
VP CAPEX (USD) & 5.264 .373 \\
VP OPEX POLÍMERO (USD) & 17.606 .113 \\
USD/Bbl & 7,29 \\
TIR (\%) & 14 \\
Payback (años) & 2,15 \\
VP OPEX MEDICIÓN VISCOSIDAD (USD) & 316.914 USD \\
\hline
\end{tabular}

Viscosímetro in-line E1. Con la implementación de un viscosímetro in-line, se garantizan medidas en tiempo real y que el monitoreo de la viscosidad de la solución polimérica sea continuo, lo que minimiza los tiempos no productivos generados en la metodología actual.

El costo relacionado al OPEX para el viscosímetro E1 fue suministrado en el sondeo de mercado y es de \$ 38.942 USD, el cual incluye: capacitación, tres visitas a campo para verificación y seguimiento del funcionamiento del instrumento. Este costo aplica para el primer mes del proyecto, posteriormente no generaría costos ya que el viscosímetro no requiere mantenimiento mensual.

En la tabla 7, se muestran los resultados obtenidos en el análisis de la alternativa de medición de viscosidad con el viscosímetro E1.

Tabla 7. Resumen resultados viscosímetro in-line E1.

\begin{tabular}{lc}
\hline VPN (USD) & $\mathbf{1 . 5 6 5 . 3 2 0}$ \\
\hline PRODUCCIÓN INCREMENTAL (Bbls) & 3.136 .367 \\
VP CAPEX & 5.264 .373 \\
VP OPEX POLÍMERO & 17.324 .601 \\
USD/Bbl & 7,20 \\
TIR (\%) & $15 \%$ \\
Payback (año) & 2,14 \\
VP OPEX MEDICIÓN VISCOSIDAD (USD) & $\$ 35.402$ \\
\hline
\end{tabular}

Viscosímetro in-line E2. El costo del OPEX para el viscosímetro E2 fue suministrado en el sondeo de mercado y es de $\$ 47.206$ USD, el cual aplica para el primer mes del proyecto, posteriormente no genera costos ya que el viscosímetro no requiere mantenimiento mensual, en el OPEX sea incluye capacitación, tres visitas a campo para verificación y seguimiento del funcionamiento del instrumento.

En la tabla 8, se muestran los resultados obtenidos en el análisis de la alternativa de medición de viscosidad con el viscosímetro E2. 
Tabla 8. Resumen resultados viscosímetro in-line E2.

\begin{tabular}{lc}
\hline VPN (USD) & $\mathbf{1 . 5 5 7 . 8 0 7}$ \\
\hline PRODUCCIÓN INCREMENTAL (Bbls) & 3.136 .367 \\
VP CAPEX & 5.264 .373 \\
VP OPEX POLÍMERO & 17.332 .114 \\
USD/Bbl & 7,20 \\
TIR (\%) & $15 \%$ \\
Payback & 2,14 \\
VP OPEX MEDICIÓN VISCOSIDAD & $\$ 42.915$ \\
\hline
\end{tabular}

En la tabla 9, se muestran una comparación de los costos generados (OPEX) con la implementación de las alternativas de monitoreo de la viscosidad del polímero a condiciones de superficie.

Tabla 9. Valor presente del OPEX de las alternativas de medición de viscosidad.

\begin{tabular}{lc}
\hline ALTERNATIVA & OPEX (USD) \\
\hline METODOLOGÍA ACTUAL & 316.914 \\
VISCOSÍMETRO E1 & 35.402 \\
VISCOSÍMETRO E2 & 42.915 \\
\hline
\end{tabular}

La gráfica ilustra un comparativo entre las alternativas de monitoreo, cabe resaltar que el OPEX comparado, únicamente incluye la implementación del viscosímetro y el análisis de monitoreo de la metodología actual.

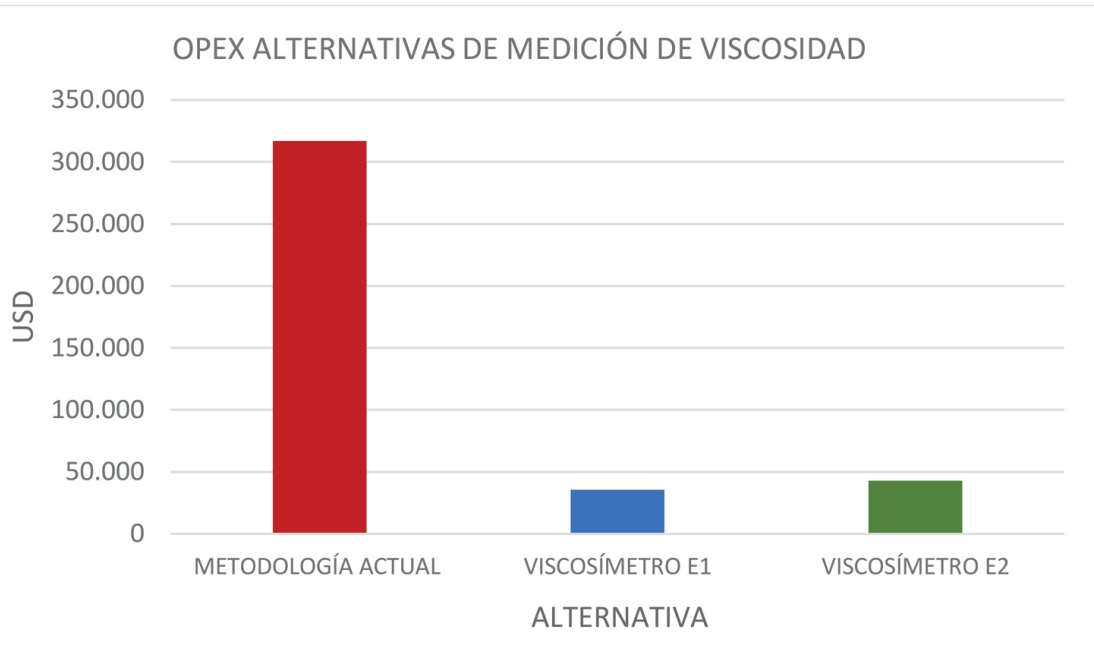

Gráfica 3. OPEX de las alternativas de monitoreo de viscosidad del polímero.

De acuerdo a los resultados financieros obtenidos se determinó que la metodología actual para la medición de viscosidad resulta ser más costosa con un valor presente de OPEX de \$316.941 USD, mientras que la alternativa más económica es la implementación del viscosímetro in-line E1 con un costo de \$35.402 USD. Sin embargo, la mejor alternativa de medición podría ser el viscosímetro in-line E2 con un costo de $\$ 42.915$
USD con el que se garantiza un proceso más confiable al ser intrínsecamente seguro.

En la tabla 10 se detallan los indicadores económicos a tener en cuenta para el análisis financiero de las alternativas de monitoreo de la viscosidad de la solución polimérica. 
Tabla 10. Indicadores económicos.

\begin{tabular}{lccc}
\cline { 2 - 4 } & VPN (USD) & TIR (\%) & Payback (Años) \\
\hline Metodología Actual & 1.351 .107 & 14 & 2,15 \\
Viscosímetro E1 & 1.565 .320 & 15 & 2,14 \\
viscosímetro E2 & 1.557 .807 & 15 & 2,14 \\
\hline
\end{tabular}

Con base a la tabla 10 , se determinó que la implementación del viscosímetro in-line es económicamente rentable, es de gran utilidad implementar esta nueva tecnología, ya que genera un beneficio en el control y monitoreo de la viscosidad de la solución polimérica durante su inyección en tiempo real.

\section{Conclusiones}

- Al emplear este sistema de medición de viscosidad in-line, se pueden tener las lecturas en tiempo real digitalizadas, almacenadas y aseguradas lo que permite tener un constante monitoreo y seguimiento de la viscosidad de la solución polimérica que es el parámetro más importante en la implementación de estos procesos de recobro.

- La implementación de estos viscosímetros inline generan una operación más segura con una transmisión digital de la información a un monitor fuera del área de operación.

- Las lecturas de los viscosímetros pueden verse afectadas por el tipo de fluido empleado y por sus condiciones de diseño, ya que las caídas de presión presentes en la línea pueden generar errores en la medición.

- Se analizaron los principios físicos de los viscosímetros in-line, teniendo en cuenta los rangos operaciones y limitaciones de cada uno de ellos, dando como resultado que los ochos viscosímetros manejan altos rangos de viscosidad por lo que pueden ser empleados en los pilotos, sin embargo, se deben tener en cuenta todos parámetros de operación para establecer el criterio de selección como lo es la presión.

- Los viscosímetros A1, C1 y D1 permiten medir otras variables de proceso tales como densidad y temperatura, opción que los demás no presentan.

- Se analizaron los ocho viscosímetros in-line con base a los rangos operacionales de los cinco pilotos de inyección de polímeros de Ecopetrol S.A determinando que la temperatura y viscosidad no son criterio de selección para los viscosímetros debido a que se encuentran dentro del rango de operación de los pilotos.
- Se determinó que los viscosímetros A2 y B1 se ajustan a los cinco pilotos, ya que manejan amplios rangos de presión.

- Se determinó que los viscosímetros A1, C1, E1 y E2 únicamente se pueden emplear en el piloto 4 debido a que sus rangos de presión son muy bajos.

- El viscosímetro F1 solo aplica para el piloto 1, ya que es el único viscosímetro que maneja presiones superiores a 2900 psi.

- El presente análisis técnico- financiero determinó que la mejor alternativa para el control y monitoreo de la viscosidad de la solución polimérica en una eventual aplicación a las condiciones del piloto 4 es el viscosímetro E2 con un OPEX menor al de la metodología actual, siendo este de $\$ 42.915$ USD y generando una diferencia de \$273.999 USD, además con su implementación se garantizan medidas en tiempo real para una mejor toma de decisiones

\section{Referencias bibliográficas}

Anton Paar. (s.f.). Manual técnico Viscosímetro en línea L-Vis 520 Ex.

Anton Paar. (2017). Viscosímetro en línea L-Vis 510. Anton Paar. Recuperado de https://www. anton-paar.com/corp-en/products/details/inlineviscometer-1-vis-510/.

Araujo, Y. C., \& Araujo, M. (2018). Polymers for application in high temperature and high salinity reservoirs-critical review of properties and aspects to consider for laboratory screening. Fuentes: El reventón energético, 16(2), 55-71.

Aya, C. L. D., Guardia, V. M. D., Toro, G. A. M., García, R. H. C., \& Pérez, H. I. Q. (2018). Metodología para la priorización de tecnologías emergentes de recobro mejorado químico. Fuentes, el reventón energético, 16(2), 55-71 
Báez, B., Montealegre, D. (2019). Análisis técnicofinanciero de las diferentes alternativas para la medición in-line de las viscosidad del polímero durante su proceso de inyección. 125.

Cruz, H. D. O., Duque, J. P. V., \& Marulanda, J. F. F. (2015). Propuesta metodológica para el control y monitoreo de un proceso de inyección de agua. Fuentes, el reventón energético, 13(2).

Ecopetrol S.A. (2018). Seguimiento a la calidad del agua del piloto de inyección.

Ecopetrol S.A. Instituto Colombiano del Petróleo. (2016). Metodologia para el diseño, ejecución, monitoreo y evaluación de procesos de recobro quimico mediante inyección de polímero. 50.

Emerson. (2017). Micro Motion Fork Viscosity Meters.

Guardia, V. M. D., Torres, M. C., Arenas, C. E. V., Castro, R. H., Toro, G. M., \& Mendoza, O. B. (2011). Análisis de riesgo y simulación de monte carlo en la valoración de proyectos-aplicación en la industria de los hidrocarburos. Fuentes, el reventón energético, 9(2).

Hydramotion Ltd. (s.f.). Ventaja XL7. Hydramotion Ltd. Recuperado de https://hydramotion. com/es/products/xl7Hydramotion Ltd. (s.f.). Especificación. Hydramotion Ltd. Recuperado de https://hydramotion.com/es/products/xl7/ specification

Jimenez, R., Castro, R., Maya, G., Pérez, R., Delgadillo, C., García, H., Cárdenas, F. (2017). Análisis comparativo de procesos de inyección de polímeros ejecutados en Colombia. ACIPET, 16.

Molano, A. M. J., Navarro, S. F. M., \& Díaz, R. J. (2014). Metodología para el diseño de baches en un proceso de inyección de polímeros para recobro mejorado, considerando fenómenos de interacción roca/fluidos. Fuentes: El reventón energético, 12(2), 6 .

Pinto, M. S., Herrera, D. M., \& Angarita, J. C. G. (2018). Production optimization for a conceptual model through combined use of polymer flooding and intelligent well technology under uncertainties. Fuentes, el reventón energético, 16(1), 37-45.

R.-H. Castro-Garcia, G.-A. Maya-Toro, R. JimenezDiaz, H.-I. Quintero-Perez, V.-M. Díaz-Guardia, K.-M. Colmenares-Vargas, et al., "Polymer flooding to improve volumetric sweep efficiency in waterflooding processes," CT\&F-Ciencia, Tecnología y Futuro, vol. 6, pp. 71-90, 2016. https://doi.org/10.29047/01225383.10

SNF Floerger. (2014). Floquip VDH: Inline Viscometer.

SNF Floerger. (2016). Geología del petróleo Sistemas petrolíferos EOR 101.

Sofraser. (s.f.). Features \& specificactions: MIVI process viscometer. Francia.

Sofraser. (2018). MIVI Process Viscometer. Francia.

Sofraser. (2015). SOFLUX: Inline viscometer for extrusion. Francia.

Sofraser. Viscosímetro de proceso MIVI 9601. Sofraser. Recuperado de http://www.viscosimetro-deproceso.es/viscosimetro-de-proceso-mivi-9601/. Francia.

VAF instruments. (2018). Technical Manual: ViscoSense3D Ex d. Países Bajos.

Fecha de recepción: 10 de Septiembre de 2019 Fecha de aceptación: 28 de octubre de 2020 